\title{
Effect of Resonant Neutrino Oscillation on TeV Neutrino Flavor Ratio from Choked GRBs
}

\author{
Sarira Sahu* and Bing Zhang ${ }^{\dagger}$ \\ * Instituto de Ciencias Nucleares, Universidad Nacional Autónoma de México, \\ Circuito Exterior, C.U., A. Postal 70-543, 04510 Mexico DF, Mexico \\ ${ }^{\dagger}$ Department of Physics and Astronomy, University of Nevada, Las Vegas, NV 89154, USA
}

\begin{abstract}
In the collapsar scenario of the long duration Gamma-Ray Bursts (GRBs), multi-TeV neutrino emission is predicted as the jet makes its way through the stellar envelope. Such a neutrino signal is also expected for more general "failed" GRBs in which a putative jet is "choked" by a heavy envelope. If the $\nu_{e} \rightarrow \nu_{\mu}$ neutrino oscillation parameters are in the atmospheric neutrino oscillation range, we show that the resonant oscillation of $\nu_{e} \leftrightarrow \nu_{\mu, \tau}$ can take place within the inner high density region of the choked jet progenitor with a heavy envelope, altering the $\nu$ flavor ratio on its surface to $\Phi_{\nu_{e}}^{s}: \Phi_{\nu_{\mu}}^{s}: \Phi_{\nu_{\tau}}^{s}=5: 11: 2$. Considering vacuum oscillation of these neutrinos on their way to Earth, the final flavor ratio detected on Earth is further modified to either $1: 1.095: 1.095$ for the large mixing angle solution to the solar neutrino data, or 1:1.3:1.3 for maximal mixing among the muon and tau neutrinos in vacuum.
\end{abstract}

PACS numbers: 98.70.Rz,96.40.Tv

\section{INTRODUCTION}

Long duration GRBs (LGRBs) are believed to be associated with deaths of massive stars [1]. The evidence in support of such an origin includes associations of several LGRBs with Type Ic supernovae and the prevalence of star forming dwarf host galaxies associated with LGRBs [2]. Observationally, only a small fraction $\left(\leq 10^{-3}\right)$ of core collapse SNe are associated with GRBs 31. They correspond to those jets that break through the stellar envelope and reach a highly relativistic speed (Lorentz factor $\Gamma \geq 100$ ). Internal shocks are formed in the optically thin regions, and gamma-rays are produced by synchrotron radiation and/or inverse-Compton scattering of Fermi accelerated electrons in these shocks.

On the other hand, it is feasible to envision that a much larger fraction of core collapses may also launch a mildly relativistic jet from the central engine, but the jet never makes its way out from the envelope, due to either a smaller energy budget or a more extended, massive stellar envelope than in a GRB progenitor. In any case, both the successful and these "choked" jets can accelerate protons to energy $\geq 10^{5} \mathrm{GeV}$ from the internal shocks well inside the stellar envelope. The interaction between these protons and the $\sim 1 \mathrm{keV}$ thermal X-ray photons emitted by the hot cocoon surrounding the jet would generate multi- $\mathrm{TeV}$ neutrinos through photopion production [4]. For an individual GRB at redshift $z \sim 1$, the predicted upward going muon event number is $0.1-10$ in a $\mathrm{km}^{3}$ detector [4]. There can also be neutrino production due to $p p$ and $p n$ collisions involving relativistic protons from the buried jet and the thermal nucleons from the jet and the surrounding, which can produce more abundant neutrinos for the presupernova stars with a heavy envelope [5]. The detection of low luminosity (LL) GRBs (such as GRB 980425 and GRB 060218 [6]) suggests that the event rate of gamma-ray dim core collapses is much higher than those associated with high luminosity GRBs
[7]. It is conceivable that the gamma-ray "dark" choked GRBs are even more abundant, and would contribute more to the high energy neutrino background.

Several other neutrino mechanisms have been discussed in the literature. The internal shocks that power the prompt gamma-ray emission can produce $\sim 100 \mathrm{TeV}$ neutrinos 8 - 10], which should be lagged behind the $\mathrm{TeV}$ neutrinos. Inelastic collisions between decoupled protons and neutrons during the acceleration of the fireball can power a multi-GeV neutrino signal [11], but the predicted flux level is below the atmospheric neutrino background, and hence, difficult to detect. The neutrinos produced in the early afterglow phase have a very high energy ( EeV) 12 and are not optimized for detection with the present day neutrino detectors. The multi-TeV neutrino signal discussed here could be above the atmospheric background, and may be detectable with $\mathrm{km}^{3}$ detectors for some nearby sources.

The main source of high energy neutrinos is the decay of charged pions, which leads to the neutrino flux ratio at the production site $\Phi_{\nu_{e}}^{0}: \Phi_{\nu_{\mu}}^{0}: \Phi_{\nu_{\tau}}^{0}=1: 2: 0\left(\Phi_{\nu_{\alpha}}^{0}\right.$ corresponds to the sum of neutrino and anti-neutrino flux for the flavor $\alpha$ at the source). The vacuum oscillation of these neutrinos on their way to Earth would make the observed ratio to $1: 1: 1$. This applies to low energy neutrinos including the $\mathrm{TeV}$ neutrinos discussed in this paper. For high energy neutrinos above $\sim 1 \mathrm{PeV}$, muon energy is degraded before decaying to low energy neutrinos so that high energy neutrinos will be absent. The neutrino flux ratio at the source is modified to $0: 1: 0$, which is further modified to $1: 1.8: 1.8$ at Earth after vacuum oscillation is taken into account [13].

Another possibility of modifying the neutrino flavor ratio is the resonant conversion of neutrinos from one flavor to another due to the medium effect. Such an effect is known to be important for solar neutrinos [14], and has been discussed for hot plasma in the early universe [15], supernova medium [17], the GRB fireball [18 20] 
and jet [21]. Here we show that for choked GRBs, the multi- $\mathrm{TeV}$ neutrinos discussed by Mészáros \& Waxman [4] could undergo resonance oscillation in the high density core (typically He core) of the presupernova star, if the neutrino oscillation parameters are in the atmospheric neutrino oscillation range. This would alter the neutrino flavor ratio escaping from the stellar envelope, and hence, the eventual detected flavor ratio from Earth.

\section{NEUTRINO OSCILLATION IN THE STELLAR ENVELOPE}

As a mildly relativistic jet makes its way through the stellar envelope, internal shocks can develop and can accelerate protons to energy $\sim 10^{5} \mathrm{GeV}$. These protons would interact with the $\sim \mathrm{keV}$ thermal X-ray photons to produce $\sim 5 \mathrm{TeV}$ neutrinos via the process $p+\gamma \rightarrow$ $\Delta^{+} \rightarrow n+\pi^{+} \rightarrow n+\mu^{+}+\nu_{\mu} \rightarrow n+e^{+}+\nu_{\mu}+\nu_{e}+\bar{\nu}_{\mu}$.

Depending on the initial mass and metalicity, the presupernova star can have different compositions with different radii. The LGRB progenitors (Type Ic SN) have lost the $\mathrm{H}$ and most of the He envelopes before explosion. They are too small to have an interesting neutrino oscillation signature. The choked jet progenitors, on the other hand, can retain the He envelope (Type Ib SNe) and even the $\mathrm{H}$ envelope (Type II SNe). These presupernova stars are favorable for $\mathrm{TeV}$ neutrino production and neutrino oscillation. It is believed that the putative jet is launched along the rotation axis where the centrifugal support is the least, and is powered by either $\nu \bar{\nu}$ annihilation or through some electromagnetic processes. Without exploring the details of jet dynamics, here we treat the jet parametrically. We assume that the jet is developed and the $\mathrm{TeV}$ neutrinos are produced at a radius $r_{\mathrm{j}} \ll R_{*}$, where $R_{*}$ is the radius of the star.

Depending on the energy of the propagating neutrino and the nature of the background, neutrinos can interact with the background particles via charge current (CC) and neutral current (NC) interactions. For a neutrino energy below $E_{\nu} \simeq M_{W}^{2} / 2 m_{e} \simeq 10^{7} \mathrm{GeV}$, an electron neutrino can have both $\mathrm{CC}$ and $\mathrm{NC}$ interactions with the normal matter, whereas muon and tau neutrinos can only have NC interactions. The effective potential of NC interactions is the same for all active neutrinos. Since oscillation depends only on the potential difference, for active-active oscillations the NC contributions cancel out. So only the CC contribution to the neutrino potential, $V=\sqrt{2} G_{F} N_{e}$, is responsible for neutrino oscillation in the medium, where $G_{F}$ is the Fermi coupling constant and $N_{e}$ is the electron number density in the medium. For anti-neutrinos $N_{e}$ is replaced by $-N_{e}$. Thus for the process $\nu_{e} \leftrightarrow \nu_{\mu, \tau}$, the neutrino potential is $\sqrt{2} G_{F} N_{e}$, while for the process $\nu_{\mu} \leftrightarrow \nu_{\tau}$ it vanishes.

Here we consider the simplified picture of two mixed flavor states $\nu_{e}$ and $\nu_{\mu}\left(\nu_{\tau}\right)$ with the vacuum mixing angle $\theta$ and mass square difference $\Delta m^{2}$. In an uniform medium the evolution of the flavor states are governed by $16,17,22]$

$$
i \frac{d}{d t}\left(\begin{array}{c}
\nu_{e} \\
\nu_{\mu}
\end{array}\right)=\left(\begin{array}{cc}
V-\Delta \cos 2 \theta & \frac{\Delta}{2} \sin 2 \theta \\
\frac{\Delta}{2} \sin 2 \theta & 0
\end{array}\right)\left(\begin{array}{l}
\nu_{e} \\
\nu_{\mu}
\end{array}\right)
$$

where $\Delta=\Delta m^{2} / 2 E_{\nu}, V$ is the potential difference between $V_{\nu_{e}}$ and $V_{\nu_{\mu}}$, (i.e. $V=V_{\nu_{e}}-V_{\nu_{\mu}}$ ) and $E_{\nu}$ is the neutrino energy. The transition probability as a function of distance $\ell$ is given by

$$
P_{\nu_{e} \rightarrow \nu_{\mu}\left(\nu_{\tau}\right)}(\ell)=\frac{\Delta^{2} \sin ^{2} 2 \theta}{\omega^{2}} \sin ^{2}\left(\frac{\omega \ell}{2}\right),
$$

with

$$
\omega=\left[(V-\Delta \cos 2 \theta)^{2}+\Delta^{2} \sin ^{2} 2 \theta\right]^{1 / 2} .
$$

Once the neutrinos are produced due to pion decay at a point $r_{\mathrm{j}} \ll R_{*}$, they will propagate away from the star where the medium effect can be substantial. If the density of the medium is such that the condition $\sqrt{2} G_{F} N_{e}=\Delta \cos 2 \theta$ is satisfied, then resonant conversion of neutrinos from one flavor to another with maximum amplitude can occur. For anti-neutrinos the resonance condition can never be satisfied (for normal neutrino mass hierarchy). Although the oscillation process $\bar{\nu}_{e} \leftrightarrow \bar{\nu}_{\mu, \tau}$ can take place, it will be suppressed.

The critical density for resonance is called the resonance density. For $5 \mathrm{TeV}$ neutrinos, it reads

$$
\rho_{\mathrm{R}}=\left(1.32 \mathrm{~g} \mathrm{~cm}^{-3}\right) \frac{\Delta \tilde{m}^{2}}{E_{\nu, 12.7}} \cos 2 \theta
$$

where we have $\Delta \tilde{m}^{2}$ in units of $\mathrm{eV}^{2}$ and $E_{\nu, 12.7}$ in units of $10^{12.7} \mathrm{eV}$. The resonance length is

$$
\ell_{\mathrm{R}}=\frac{2 \pi}{\Delta \sin 2 \theta}=1.24 \times 10^{9} \mathrm{~cm}\left(\frac{E_{\nu, 12.7}}{\Delta \tilde{m}^{2}}\right) \frac{1}{\sin 2 \theta} .
$$

Define the stellar radius $r_{\mathrm{R}}$ as the radius at which the local density is $\rho_{\mathrm{R}}$. The first condition for resonant oscillation is $\ell_{\mathrm{R}}<r_{\mathrm{R}}$.

If the resonance region is wide enough the transition can be total. We can define a resonance width for which the amplitude of the probability can be $1 / 2$ instead of unity. In this case the width can be given as $\Gamma=2 \Delta m^{2} \sin 2 \theta$. This corresponds to a length scale

$$
\delta r_{\mathrm{R}}=\frac{2 \tan 2 \theta}{\left|\frac{1}{N_{e}} \frac{d N_{e}}{d r}\right|_{\mathrm{R}}} .
$$

For $\delta r_{\mathrm{R}}>\ell_{\mathrm{R}}$, there can be enough time for $\nu_{e}$ to stay in the resonance region to convert into $\nu_{\mu}\left(\nu_{\tau}\right)$. This is the second condition for significant resonant oscillation.

In order to evaluate both conditions, one needs to know the matter density profile in the stars (which determines $r_{\mathrm{R}}$ and $\left.d N_{e} / d r\right)$. The density profile of a presupernova star is difficult to probe observationally. Numerical models predict a decreasing density with radius. If convective 
mixing is not important, there is a sharp decrease in density beyond the He core with radius $r_{\mathrm{He}} \sim 10^{11} \mathrm{~cm}$ and the local density $\rho_{\mathrm{He}} \sim 10^{-3} \mathrm{~g} \mathrm{~cm}^{-3}$. If convective mixing is important, there is no abrupt transition, and the density profile may be roughly described in the analytical form [5, 23]

$$
\rho(r)=\rho_{0}\left(\frac{R_{*}}{r}-1\right)^{n} .
$$

The parameters $R_{*}$ and $\rho_{0}$ depend on the type of the star. For example, a blue supergiant (BSG) model for SN $1987 \mathrm{~A}$ gives $R_{*}=3 \times 10^{12} \mathrm{~cm}$ and $\rho_{0}=3 \times 10^{-5} \mathrm{~g} \mathrm{~cm}^{-3}$ [24]. In some models, the He core can extend to $10^{12} \mathrm{~cm}$,

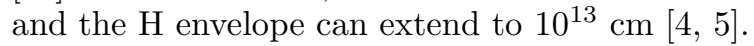

The exponent $n=3,3 / 2$ correspond to the radiative and convective envelope, respectively. In general it can vary between 2 and 3 for different numerical models. The condition $\delta r_{R}>\ell_{\mathrm{R}}$ can be re-written as a requirement to $n$ (where $r \sim \ell_{\mathrm{R}}$ has been adopted which is relevant for resonant oscillation)

$$
n<2 \tan 2 \theta\left(1-\frac{\ell_{\mathrm{R}}}{R_{*}}\right) .
$$

The resonance length $\ell_{R}$ depends on neutrino oscillation parameters and neutrino energy. Apparently, if $\ell_{\mathrm{R}} \geq$ $R_{*}$, the requirement to $n(n<0)$ is unphysical, and no neutrino oscillation is expected inside these stars. On the other hand, if $\ell_{\mathrm{R}} \ll R_{*}$, the constraint $n<2 \tan 2 \theta$ may be satisfied in some stars for some oscillation parameters.

In order to evaluate whether the neutrino oscillation conditions are satisfied, one needs to know the neutrino oscillation parameters in matter. Experimentally these are inaccessible. Only oscillation parameters from the solar and atmospheric neutrino experiments are available. For small neutrino mixing angles, the mixing matrix is almost diagonal and each flavor eigenstate nearly overlaps with one of the mass eigenstate. One may then associate $\nu_{e}$ to $\nu_{1}, \nu_{\mu}$ to $\nu_{2}$, and $\nu_{\tau}$ to $\nu_{3}$. While the solar neutrino oscillation parameters are relevant to $\nu_{e} \rightarrow \nu_{\mu}$ oscillations, the atmospheric neutrino oscillation data correspond mostly to $\nu_{\mu} \rightarrow \nu_{\tau}$ oscillations, and the corresponding neutrino parameters are $\theta_{23}$ and $\Delta m_{23}^{2}$, respectively. It is also very possible that the physical properties of neutrinos in a medium could be different from their vacuum values. For example, a neutrino can acquire mass due to its interaction with the background particles even if we consider it massless in vacuum. Similarly the mixing properties in matter may not follow the vacuum pattern as measured. Nonetheless, since the oscillation parameters of the solar and atmospheric experiments are the best measured, in the following we test whether these parameters may allow neutrino oscillation to happen in the progenitor stars of choked GRBs. We do not take it for granted that any of these parameters are operating in the oscillation process $\nu_{e} \leftrightarrow \nu_{\mu, \tau}$ in the choked fireball, but just take the only experimentally available parameters to test the conditions for prominent oscillations. Similar analyses have been carried out before to evaluate the possible oscillation effect in GRB fireballs [18, 19].

The SNO salt phase solar neutrino data, combined with the KamLand reactor antineutrino results, constrain the neutrino oscillation parameters in the regime $6 \times$ $10^{-5} \mathrm{eV}^{2}<\Delta m^{2}<10^{-4} \mathrm{eV}^{2}$ and $0.64<\sin ^{2} 2 \theta<0.96$ [25], with the best fit parameters $\Delta m^{2} \sim 7.1 \times 10^{-5} \mathrm{eV}^{2}$ and $\sin ^{2} 2 \theta \sim 0.69$ with $99 \%$ confidence level. The best fit values give $\rho_{\mathrm{R}, \mathrm{SNO}} \simeq 5.2 \times 10^{-5} \mathrm{~g} \mathrm{~cm}^{-3} E_{\nu, 12.7}^{-1}$ and $\ell_{\mathrm{R}, \mathrm{SNO}} \simeq 2.1 \times 10^{13} \mathrm{~cm} E_{\nu, 12.7}$. We can see that $\ell_{\mathrm{R}}$ is larger than $R_{*}$ of a typical BSG, suggesting that resonant oscillation would not occur for these neutrino oscillation parameters.

On the other hand, the atmospheric neutrino oscillation parameters reported by the SK Collaboration are in the range $1.9 \times 10^{-3} \mathrm{eV}^{2}<\Delta m^{2}<3.0 \times 10^{-3} \mathrm{eV}^{2}$ and $0.9 \leq \sin ^{2} 2 \theta \leq 1.0$ at a $90 \%$ confidence level [26], which corresponds to the oscillations of mostly muon neutrinos to tau neutrinos. If we assume that these parameters apply to $\nu_{e} \rightarrow \nu_{\mu, \tau}$ oscillations in matter, we can get the following constraint. We consider the good fit point $\Delta m^{2} \sim 2.5 \times 10^{-3} \mathrm{eV}^{2}$ and $\sin ^{2} 2 \theta \sim 0.9$, and get $\rho_{\mathrm{R}, \mathrm{SK}} \simeq 1.0 \times 10^{-3} \mathrm{~g} \mathrm{~cm}^{-3} E_{\nu, 12.7}^{-1}$ and $\ell_{\mathrm{R}, \mathrm{SK}} \simeq$ $5.2 \times 10^{11} \mathrm{~cm} E_{\nu, 12.7}$. For the nominal BSG model discussed in this paper, the stellar radius at which the density is $\rho_{\mathrm{R}, \mathrm{SK}}$ is $r_{\mathrm{R}} \simeq 7.1 \times 10^{11} \mathrm{~cm}$ for $n=3$ and $E_{\nu, 12.7}=1$. We can see that the condition $\ell_{\mathrm{R}}<r_{\mathrm{R}}$ is satisfied for the typical neutrino energy $E_{\nu}=5 \mathrm{TeV}$. By using the value of $\tan 2 \theta$ from the SK neutrino data and $\ell_{\mathrm{R}}=\ell_{\mathrm{R}, \mathrm{SK}}$, we obtain from Eq. (8) $n<4.96$. Known stellar models have $n$ between 2 and 3. This suggests that the second condition is also fully satisfied. We conclude that resonant oscillation of multi- $\mathrm{TeV}$ neutrinos can occur within a nominal BSG progenitor for the neutrino oscillation parameters inferred by the atmospheric neutrino data. A similar analysis suggests that the same conclusion applies to other BSG progenitors or He stars with extended envelopes (with $R_{*}$ up to $10^{12} \mathrm{~cm}$ ), but does not apply to typical He stars (with $R_{*}=10^{11} \mathrm{~cm}$ ), or other more compact stars. Since GRB observations favor associations with Type Ic SNe (for which the He envelope is mostly stripped off), GRBs are not preferred sources for $\mathrm{TeV}$ neutrino oscillation. Instead, we identify choked GRBs, especially those with a heavy envelope, as interesting sources for resonant neutrino oscillation.

For a full oscillation, $\nu_{e}$ can oscillate to $\nu_{\mu}$ and to $\nu_{\tau}$ with equal probability but $\nu_{\mu}$ can oscillate only to $\nu_{e}$. On average we can have $1 / 3$ survival probability for $\nu_{e}$, $\nu_{\mu}$ and $\nu_{\tau}$ for each $\nu_{e}$ oscillation, but have $1 / 2$ survival probability for $\nu_{e}, \nu_{\mu}$ for the $\nu_{\mu} \leftrightarrow \nu_{e}$ resonant oscillation. The $p \gamma$ process also produce $\bar{\nu}_{\mu}$ which does not resonantly oscillate. Putting together, on the surface of the presupernova star the survival probability of each flavor (both neutrino and anti-neutrino) is in the ratio of $\left(\frac{1}{3}+\frac{1}{2}\right):\left(\frac{1}{3}+\frac{1}{2}+1\right): \frac{1}{3}=5: 11: 2$. Above $r_{R}$ (mostly in the $\mathrm{H}$ envelope), the density is much lower than the resonance density, and no further reconversion of neutrinos can take place. So the ratio $5: 11: 2$ is final neutrino 
flavor ratio escaping from the star. This ratio is notably different from the nominal $1: 2: 0$ ratio for multi-TeV neutrinos without considering the resonant oscillation effect.

\section{NEUTRINO OSCILLATION IN VACUUM}

Since these choked GRB neutrino sources are typically at large distances, the $\mathrm{TeV}$ neutrinos escaping from the star would undergo vacuum oscillation on their way to Earth. The neutrino flux for a particular flavor $\alpha$ on Earth is given by

$$
\Phi_{\nu_{\alpha}}=\sum_{\beta} P_{\alpha \beta} \Phi_{\nu_{\beta}}^{s},
$$

where $\Phi_{\nu_{\beta}}^{s}$ signifies the flux of $\nu_{\beta}$ at the surface of the He envelope after resonant oscillation, and $P_{\alpha \beta}$ corresponds to the oscillation probability from $\nu_{\alpha}$ to $\nu_{\beta}$ in vacuum. For the matter effect on resonant oscillation in the stellar envelope, we have applied a two-flavor neutrino analysis. This is because no $\nu_{\tau}$ 's are generated in the $p \gamma$ process on one hand, and the two-flavor neutrino analysis is simpler on the other hand, as it depends only on one mass square difference. The limited size of the oscillation baseline (the stellar envelope) also makes the three-flavor oscillation effect unimportant. Such a two-flavor neutrino analysis has been applied in most previous resonant oscillation discussions [18, 22].

When discussing the vacuum oscillation effect along a long base line from the source to Earth, one needs however fully take into account the three-flavor neutrino oscillation effect. This is demanded by the combined analyses of both the solar and the atmospheric neutrino anomalies. For the best fit to the SNO data from the large mixing angle (LMA) solution one can take the mixing angles $\theta_{12}=34^{\circ} \pm 2.5^{\circ}, \theta_{23}=45^{\circ} \pm 6^{\circ}, \theta_{13}=0^{\circ} \pm 8^{\circ}$ and the Dirac phase $\delta=0[13,27]$. This gives $P_{e e} \simeq 0.57$, $P_{e \mu}=P_{e \tau} \simeq 0.215$ and $P_{\mu \mu}=P_{\mu \tau}=P_{\tau \tau} \simeq 0.393$. Inserting these probabilities in Eq. (9) and considering the error of $\theta_{12}$, the flux ratio at Earth is $1:(1.095 \pm 0.012)$ : $(1.095 \pm 0.012)$. On the other hand, if we consider the maximal mixing among the $\nu_{\mu}$ and $\nu_{\tau}$ in vacuum, then the $\nu_{e}$ oscillation to $\nu_{\mu}$ and $\nu_{\tau}$ is largely suppressed. One can then have $P_{e e} \simeq 1, P_{\mu \mu}=P_{\tau \tau}=P_{\mu \tau}=1 / 2$, and all other transition probabilities are negligible. Using these oscillation probabilities, we obtain the flux ratio at Earth as $1: 1.3: 1.3$.

\section{DISCUSSION}

GRBs have a wide redshift distribution (from $z=$ 0.0085 to $z=8.2$ ). Observations suggest that the nearby low-luminosity GRBs have a local event rate $\sim 100$ time higher than that of high-luminosity GRBs [7]. If GRB jets become progressively successful in progressively rarer progenitors, it is conceivable that there could be even more choked GRBs in the nearby universe. Although the gamma-ray luminosity becomes progressively smaller as the envelope becomes progressively heavier, the TeV neutrino luminosity may not decrease, and could even follow an opposite trend. Assuming that the choked GRB progenitor has a local event rate similar to that of LL-GRBs, i.e. $\mathcal{R} \sim 200 \mathrm{Gpc}^{-3} \mathrm{yr}^{-1}$, one would expect $\sim 14$ neutrino bursts (without gamma-ray counterpart) per year all sky at $z<0.1$. If the $\mathrm{TeV}$ neutrino luminosity of these events are similar to those of successful GRBs [4, [5], then each event would have $100 \mathrm{~s}$ of $\mathrm{TeV}$ neutrinos detected by a $\mathrm{km}^{3}$ detector such as IceCube. Such a possibility is already ruled out by the current upper limits placed by the IceCube observations. This suggests that either there are not that many nearby choked GRBs, or that the choked GRBs are not as neutrino-luminous as predicted [4]. Going to the conservative extreme, i.e. if the neutrino luminosity is correlated with gamma-ray luminosity, then the detected event rate for these nearby neutrino burst sources would be 0.001-0.01 neutrinos per event in a $\mathrm{km}^{3}$ detector. This is essentially impossible for IceCube to detect individual sources. The real detected neutrino event rate may be between these two extreme values. IceCube or a similar detector would be able to detect these neutrino bursts or to place even more stringent upper limits in the near future.

If the nearby neutrino bursts are bright enough, the deviation of the observed neutrino flavor ratio from 1:1:1 may be tested by IceCube or similar detectors. The flavor ratios can be in principle deduced from the relative rates of showers, muon tracks, and the unique tau lepton induced signals [28]. The possibility of detecting a tau signal by IceCube is low, especially in the multi$\mathrm{TeV}$ energy range. On the other hand, IceCube can distinguish between shower-like events and the $\mu$-track events, although it is hard to identify $\nu_{e}$ and $\nu_{\tau}$ through their electromagnetic and hadronic showers. Nonetheless, assuming a flavor-independent neutrino spectrum and $\nu_{\mu}-\nu_{\tau}$ symmetry (as is the case of our two predicted ratios), $\nu_{e}$ fraction may be extracted from the measured Muon to shower ratio [28]. The 10\% difference in the flavor ratio reduces the $\nu_{e}$ fraction from $1 / 3$ to 0.313 (for flavor ratio 1:1.095:1.095). This corresponds to a slight increase of Muon to shower ratio. With the uncertainty $(20 \%)$ for the nominal diffuse flux $\left(E_{\nu_{\mu}}^{2} d N_{\nu_{\mu}} / d E_{\nu_{\mu}}=10^{-7} \mathrm{GeV} \mathrm{cm}^{-2} \mathrm{~s}^{-1}\right.$ for one year) adopted in [28], the small change in Muon to shower ratio may not be differentiated. If nearby neutrino bursts are bright enough, the flux would be increased and the uncertainty reduced significantly. This would make a better case for detecting the flavor ratio change. For the $1: 1.3: 1.3$ ratio, the $\nu_{e}$ fraction is reduced from $1 / 3$ to 0.28 , making the Muon to shower ratio as high as $\sim 3.5$ (as compared to $\sim 3$ for $1: 1: 1)$. The effect may be detectable for the putative bright neutrino burst events discussed above, even if they may be very rare.

Since the parameters $\left(\ell_{\mathrm{R}}, \rho_{\mathrm{R}}, r_{\mathrm{R}}\right.$, and $\left.\delta r_{\mathrm{R}}\right)$ all de- 
pend on $E_{\nu}$, we expect the flavor ratio would also depend on neutrino energy. This aspect has been extensive discussed in [29].

This work is partially supported by DGAPA-UNAM
(Mexico) Project No. IN101409 and Conacyt project No. 103520 (SS) and by NASA NNX09AO94G and NSF AST-0908362 (BZ).
[1] S. E. Woosley, Astrophys. J. 405, 273 (1993); B. Paczyński, Astrophys. J. 494, L45 (1998).

[2] S. E. Woosley and J. S. Bloom, Ann. Rev. Astron. Astrophys. 44, 507 (2006); A. S. Fruchter, et al., Nature 441, 463 (2006); Zhang, B. et al., Astrophys. J. 703, 1696 (2009).

[3] E. Berger, S. R. Kulkarni, D. A. Frail and A. M. Soderberg, Astrophys. J. 599, 408 (2003);

[4] P. Mészáros and E. Waxman, Phys. Rev. Lett. 87, 171102 (2001).

[5] S. Razzaque, P. Mészáros and E. Waxman, Phys. Rev. Lett. 93, 181101 (2004) [Erratum-ibid. 94, 109903 (2005)]; S. Razzaque, P. Mészáros and E. Waxman, Phys. Rev. D 69, 023001 (2004).

[6] T. J. Galama et al., Nature, 395, 670 (1998); S. Campana et al., Nature 442, 1008 (2006).

[7] E. Liang et al., Astrophys. J. 662, 1111 (2007); K. Murase et al., Astrophys. J. 651, L5 (2006); N. Gupta and B. Zhang, AstroParticle. Phys. 27, 386 (2007); F. Virgili et al., Mon. Not. R. Astron. Soc., 392, 91 (2009).

[8] E. Waxman and J. N. Bahcall, Phys. Rev. Lett. 78, 2292 (1997); E. Waxman and J. N. Bahcall, Phys. Rev. D 59, 023002 (1999).

[9] X. Y. Wang and Z. G. Dai, Astrophys. J. Lett. 691, L67 (2009) arXiv:0807.0290 [astro-ph]].

[10] K. Murase, Phys. Rev. D 78, 101302 (2008) arXiv:0807.0919 [astro-ph]].

[11] J. N. Bahcall and P. Mészáros, Phys. Rev. Lett. 85, 1362 (2000); P. Mészáros and M. J. Rees, Astrophys. J. 541, L5 (2000).

[12] E. Waxman and J. N. Bahcall, Astrophys. J. 541, 707 (2000); Z. G. Dai and T. Lu, Astrophys. J. 551, 249 (2001).

[13] T. Kashti and E. Waxman, Phys. Rev. Lett. 95, 181101 (2005).

[14] S. P. Mikheyev and A. Yu. Smirnov, Yad. Fiz. 42, 1441
(1985).

[15] K. Enqvist, K. Kainulainen and J. Maalampi, Nucl. Phys. B 349, 754 (1991).

[16] C. W. Kim and A. Pevsner, Neutrinos in Physics and Astrophysics, Harood Academic Publishers, 1993, Switzerland, see pages 174-176.

[17] S. Sahu and V. M. Bannur, Phys. Rev. D 61, 023003 (2000).

[18] S. Sahu and J. C. D'Olivo, Phys. Rev. D 71, 047303 (2005);

[19] S. Sahu, N. Fraija and Y. Y. Keum, Phys. Rev. D 80, 033009 (2009).

[20] S. Sahu, N. Fraija and Y. Y. Keum, JCAP 11, 024 (2009).

[21] O. Mena, I. Mocioiu and S. Razzaque, Phys. Rev. D 75, 063003 (2007).

[22] M. C. Gonzalez-Garcia and Y. Nir, Rev. Mod. Phys. 75, 345 (2003).

[23] C. D. Matzner and C. F. McKee, Astrophys. J. 510, 379 (1999); E. Waxman and P. Mészáros, Astrophys. J. 584, 390 (2003).

[24] T. Shigeyama and K. Nomota, Astrophys. J. 360, 242 (1990); D. Arnett, Astrophys. J. 383, 295 (1991).

[25] S. N. Ahmed et al. [SNO Collaboration], Phys. Rev. Lett. 92, 181301 (2004); T. Araki et al. [KamLAND Collaboration], Phys. Rev. Lett. 94, 081801 (2005).

[26] Y. Ashie et al. [Super-Kamiokande Collaboration], Phys. Rev. Lett. 93, 101801 (2004).

[27] A. Strumia and F. Vissani, Nucl. Phys. B 726, 294 (2005).

[28] J. F. Beacom, N. F. Bell, D. Hooper, S. Pakvasa and T. J. Weiler, Phys. Rev. D 68, 093005 (2003) [Erratumibid. D 72, 019901 (2005)].

[29] S. Razzaque and A. Y. Smirnov, JHEP 1003, 031 (2010) arXiv:0912.4028 [hep-ph]]. 